\title{
White matter lesions and intra-arterial thrombolysis
}

\author{
Simon Jung $\cdot$ Marie Luise Mono $\cdot$ Oliver Findling $\cdot$ Urs Fischer $\cdot$ Aekaterini Galimanis $\cdot$ \\ Anja Weck · Gian Marco De Marchis • Pietro Ballinari · Jan Gralla · Caspar Brekenfeld • \\ Gerhard Schroth $\cdot$ Marcel Arnold $\cdot$ Heinrich P. Mattle $\cdot$ Marwan El-Koussy
}

Received: 25 August 2011/Revised: 18 November 2011/ Accepted: 1 December 2011/Published online: 17 January 2012

(C) Springer-Verlag 2012

\begin{abstract}
The aim of the study was to assess the influence of white matter lesions in patients with acute ischemic stroke treated with intra-arterial thrombolysis (IAT). From September 2003 to January 2010, we treated 400 patients with IAT at our institution. Of these patients, 292 were evaluated with MRI scans and included in this observational study. Clinical data were collected prospectively. Outcome after 3 months was measured with the modified Rankin Scale (mRS); mRS 0-1 was considered as favorable outcome. White matter lesions were scored visually by two observers using the semiquantitative Scheltens and Fazekas scores. Logistic regression analysis was used to identify the association of white matter lesions and clinical outcome, recanalization, and cerebral hemorrhage. The severity of white matter lesions was inversely correlated with favorable outcome, survival and successful recanalization. White matter lesions were an independent predictor of outcome (OR $0.569, p=0.007$ ) and survival (OR 0.550, $p=0.018$ ) and a weak but independent predictor for recanalization (OR $0.949, p=0.038)$. Asymptomatic intracerebral bleeding after IAT was associated with white matter lesions in the basal ganglia in the univariate analysis $(p=0.036)$, but not after multivariable analysis. The severity of white matter lesions independently predicts clinical outcome and survival
\end{abstract}

S. Jung · M. L. Mono · O. Findling · U. Fischer .

A. Galimanis · A. Weck · G. M. De Marchis · P. Ballinari ·

M. Arnold · H. P. Mattle $(\bowtie)$

Department of Neurology, Inselspital, University Hospital Bern,

University of Bern, Freiburgstrasse 10, 3010 Bern, Switzerland

e-mail: heinrich.mattle@insel.ch

J. Gralla · C. Brekenfeld · G. Schroth · M. El-Koussy

Department of Neuroradiology, Inselspital, University Hospital

Bern, University of Bern, Freiburgstrasse 10,

3010 Bern, Switzerland in patients treated with IAT. White matter lesions are also a weak but independent predictor for recanalization. Symptomatic intracranial bleeding after IAT are not associated with white matter lesions. Therefore, white matter lesions should not be considered as a contraindication against IAT.

Keywords White matter lesions - Leukoaraiosis . Intra-arterial · Thrombolysis · Outcome

\section{Introduction}

Lesions that comprise periventricular, deep and subcortical white matter attracted great attention since French reports in the nineteenth century and since Otto Binswanger's German report in 1894. The advent of cerebral imaging rekindled this interest, first the hypodensities of the white matter seen on computed tomography and later the impressive hyperintensities visible on T2-weighted magnetic resonance imaging (MRI). Today, we know that the periventricular, deep and subcortical T2-hyperintensities on MRI are nonspecific. Therefore, white matter lesions have also received the term leukoaraiosis. White matter lesions are a sign of ischemic damage due to various small vessel diseases, dilated perivascular spaces, or lacunar infarcts. Several factors were identified that influence the severity of these white matter lesions. The strongest association was found for increasing age and hypertension [1,2]. Other factors such as diabetes mellitus, atherosclerosis, hyperlipidemia, hyperhomocysteinemia, and smoking were also identified as risk factors, but with weaker associations. Furthermore, genetic background seems to play a role. The distribution of white matter lesions within the brain is not even; in general white matter lesions are more pronounced in anterior compared to posterior brain regions [3]. 
White matter lesions represent an independent risk factor for stroke [4] and predict unfavorable outcome after stroke [5]. There is a major concern that white matter lesions affect outcome in patients with acute ischemic stroke: three studies showed that patients with white matter lesions have a greater risk of intracranial hemorrhage after intravenous thrombolysis [6-8]. Because of the association with outcome and bleeding complications, white matter lesions have become an issue for treating physicians. The aim of the present study was to evaluate the predictive value of white matter lesions and their regional distribution for outcome and complications of IAT for acute ischemic stroke.

\section{Patients and methods}

From September 2003 to January 2010, 400 patients were treated with IAT for acute stroke in our Stroke Unit. Patients who were examined with MRI scans of the brain within 30 days from stroke onset were selected for this study $(n=292)$ but not patients examined with CT scans $(n=103)$ or inadequate MR image quality $(n=5)$.

\section{Clinical data}

All patients were examined immediately after admission by a neurologist and the neurologic deficit was scored using the National Institutes of Health Stroke Scale (NIHSS) [9]. Demographic data, time of symptom onset, and previous medical history (prestroke handicap, history of coronary artery disease, atrial fibrillation, transient ischemic attack (TIA) ischemic stroke) and stroke risk factors such as sex, hypertension, diabetes, current cigarette smoking, hypercholesterolemia, coronary heart disease, and a family history of TIA and stroke were recorded. Digital substraction angiography was performed via a transfemoral approach using a biplane, high-resolution angiography system. All patients underwent complete four-vessel cerebral angiography to assess vessel occlusions and collateral flow. The site of artery occlusion was categorized as follows: proximal and distal internal carotid artery, carotid-T, M1/M2/ M3-4 segments of the middle cerebral artery, A1/A2 segments of the anterior cerebral artery, P1/P2 segments of the posterior cerebral artery, proximal/mid/distal segments of the basilar artery, and the vertebral artery. Collaterals were graded as reported previously [10].

The interventional neuroradiologists decided on the use of urokinase, mechanical intervention, or both as recanalization techniques. At the end of the angiography, recanalization was classified according to thrombolysis in myocardial infarction (TIMI) grades [11]: 1, minimal recanalization; 2, partial and 3 , complete recanalization.
A computed tomography (CT) or MRI scan was obtained to assess bleeding complications 24-72 $\mathrm{h}$ after treatment. Symptomatic ( $\mathrm{SICH}$ ) and asymptomatic intracerebral bleedings (aICH) were scored according to the PROACT II Study [12].

The etiology of the stroke was classified according to the TOAST criteria using ancillary investigations as necessary [13]. Secondary preventive treatment was given according to European guidelines [14, 15].

Medium-term outcome was assessed 3 months after stroke using the modified Rankin scale (mRS) [16]. The follow-up information was obtained by clinical examinations in $78.5 \%$ of the patients or by structured telephone interviews in $21.5 \%$.

Imaging methods

MRI was performed using a $1.5 \mathrm{~T}$ MR imaging system (Magnetom, Siemens). We used axial T1 (TR 2,000 ms, TE $40 \mathrm{~ms}$ ), T2 (TR 2,000 ms, TE $50 \mathrm{~ms}$ ), proton density (TR 5,000 ms, TE $96 \mathrm{~ms}$ ), and diffusion-weighted imaging (TR 3,200 ms, TE $87 \mathrm{~ms}$ ), with automatic calculation of the apparent diffusion coefficient (ADC maps).

Imaging analysis

Slice thickness was $5 \mathrm{~mm}$. White matter lesions were scored using two different visual scoring systems. With the scoring system of Fazekas and Schmidt [17] periventricular hyperintensities (Fazekas PV) were graded as follows: if absent as 0 , caps or pencil-thin lining as 1 , smooth halo as 2 , and irregular periventricular white matter lesions extending into the deep white matter as 3. Subcortical and deep white matter lesions (Fazekas WM) were scored as follows: absent as 0 , punctate foci as 1 , a beginning confluence of foci as 2, and large confluent areas as 3 . The second scoring system used was the Scheltens score [18]. The periventricular region (Scheltens PV) was divided into the frontal caps, occipital caps and the bands in between the caps. Each region was separately scored as follows: absent as 0 , white matter lesions up to $5 \mathrm{~mm}$ as 1 and larger than $5 \mathrm{~mm}$ as 2 . Accordingly, the deep white matter (Scheltens WM) was divided into frontal, parietal, occipital, and temporal regions. The basal ganglia (Scheltens $B G$ ) were divided into caudate nucleus, putamen, globus pallidus, thalamus, and internal capsule. Each of these regions was scored as follows: absent as $0,<6$ lesions with a diameter smaller than $3 \mathrm{~mm}$ as $1,>5$ lesions with a diameter smaller than $3 \mathrm{~mm}$ as $2,<6$ lesions with a diameter of $4-10 \mathrm{~mm}$ as $3,>5$ lesions with a diameter of $4-10 \mathrm{~mm}$ as 4 , at least one lesion $>10 \mathrm{~mm}$ diameter as 5, and confluent lesions as 6 .

Scores for Scheltens PV, WM, and BG were calculated by summation of the different regions. All MRI scans were 
scored jointly by two examiners (S.J. and M. E.-K.) blinded to clinical data. Whenever they scored differently, the grade was decided by consensus.

\section{Statistical analysis}

Statistical analysis was performed using SPSS 18 (SPSS Inc., Chicago, IL, USA). Forward stepwise logistic regression analysis was carried out on the predictive factors identified by univariate analysis $\left[\chi^{2}\right.$ test or for continuous variables Mann-Whitney test with $p<0.2$; considering age, gender, time to thrombolysis, NIHSS score on admission, TOAST classification, diabetes mellitus, hypertension, hypercholesterolemia, atrial fibrillation, artery dissection, coronary artery disease, previous stroke, previous use of antithrombotic therapy, smoking, family history of stroke, occlusion type, collaterals, TIMI grade after therapy, Fazekas sum score, Scheltens sum score, and scores of the subregions (PV, WM, BG)]. Predictors of outcome were analyzed by comparing patients with a favorable clinical outcome (mRS 0-1) and those with a less favorable outcome (mRS 2-6). Predictors of survival were analyzed by comparing stroke survivors (mRS 0-5) and patients who died (mRS 6) within 3 months after the stroke. Predictors of recanalization were analyzed by comparing poor (TIMI 0-1) and good (TIMI 2-3) recanalization. Predictors of intracerebral bleeding after thrombolysis were analyzed by comparing patients with sICH or aICH and those without hemorrhage.

\section{Results}

A total of 292 patients had MRI scans within 30 days from stroke onset and were included in this study; 229 (78.4\%) of the 292 MRI scans were obtained on the day of symptom onset, 63 (21.6\%) within 26 days of stroke onset.

The baseline characteristics, treatment, outcome, and complications are summarized in Table 1. Twenty-eight (9.6\%) of the 292 patients were older than 80 years. Followup was obtained from 289 patients (99\%), from 227 (77.7\%) by clinical examination, and from $62(22.3 \%)$ by phone interview. Prior to stroke, 291 patients were independent.

Results of white matter lesion scoring with the Fazekas and Scheltens scores in relation to outcome are given in Table 2. Mean values of the sum scores of each brain region are given.

Predictors of outcome and survival

In multivariate logistic regression analysis lower age, lower baseline NIHSS, shorter time to treatment, absence of diabetes mellitus, good collaterals as seen on digital
Table 1 Baseline characteristics, treatment, outcome, and complications of 292 patients with acute ischemic stroke

\begin{tabular}{|c|c|}
\hline & $N(\%)^{\mathrm{a}}$ \\
\hline \multicolumn{2}{|l|}{ Baseline characteristics and treatment } \\
\hline Age, years (standard deviation) & $64(15)$ \\
\hline Female sex & $161(44.9)$ \\
\hline \multicolumn{2}{|l|}{ Vascular risk factors } \\
\hline Hypertension & $171(58.6)$ \\
\hline Diabetes mellitus & $45(15.4)$ \\
\hline Current smoking $(n=290)$ & $65(22.4)$ \\
\hline Hypercholesterolemia & $161(55.1)$ \\
\hline Coronary artery disease & $43(14.7)$ \\
\hline Atrial fibrillation $(n=290)$ & $86(29.7)$ \\
\hline Previous stroke & $20(6.8)$ \\
\hline Family history of stroke $(n=281)$ & $39(13.9)$ \\
\hline Previous ASS/OAC & $100(34.2)$ \\
\hline \multicolumn{2}{|l|}{ Stroke etiology } \\
\hline Large artery disease & $58(19.9)$ \\
\hline Cardioembolic & $126(43.2)$ \\
\hline Other determined etiology & $37(12.7)$ \\
\hline Unknown etiology & $70(23.9)$ \\
\hline Dissection & $12(4.1)$ \\
\hline Baseline NIHSS score, median (range) & $15(2-36)$ \\
\hline \multicolumn{2}{|l|}{ Occlusion localization } \\
\hline Internal carotid artery & $44(15.1)$ \\
\hline Carotid $\mathrm{T}$ & $28(9.6)$ \\
\hline Anterior cerebral artery & $1(0.3)$ \\
\hline Middle cerebral artery & $160(4.8)$ \\
\hline Posterior cerebral artery & $3(1.0)$ \\
\hline Basilar artery & $55(18.8)$ \\
\hline Vertebral artery & $1(0.3)$ \\
\hline \multicolumn{2}{|l|}{ Intraarterial thrombolysis type } \\
\hline Urokinase only & $237(1.2)$ \\
\hline Bridging IVT and IAT & $9(3.1)$ \\
\hline Mechanical only & $46(15.8)$ \\
\hline $\begin{array}{l}\text { Minutes from symptom onset to treatment, } \\
\text { median (range) }\end{array}$ & $286(82-1,440)$ \\
\hline Urokinase dose, median IE (range) & $\begin{array}{l}1,000,000 \\
(0-1,500,000)\end{array}$ \\
\hline \multicolumn{2}{|l|}{ Outcome parameters } \\
\hline mRS $0-1(n=289)$ & $76(26.3)$ \\
\hline Survival $(n=289)$ & $226(77.4)$ \\
\hline \multicolumn{2}{|l|}{ Recanalization grade } \\
\hline TIMI 0 & $59(20.2)$ \\
\hline TIMI 1 & $19(6.5)$ \\
\hline TIMI 2 & $58(19.9)$ \\
\hline TIMI 3 & $156(53.4)$ \\
\hline \multicolumn{2}{|l|}{ Bleeding complications } \\
\hline $\mathrm{sICH}$ & $12(4.1)$ \\
\hline $\mathrm{aICH}$ & $70(24.0)$ \\
\hline
\end{tabular}

\footnotetext{
a Number and percent of patients unless stated otherwise
} 
Table 2 Mean white matter lesion scores and outcome, two-tailed significance levels in brackets

\begin{tabular}{|c|c|c|c|c|c|c|c|}
\hline & Fazekas & Scheltens & $\begin{array}{l}\text { Fazekas peri- } \\
\text { ventricular }\end{array}$ & $\begin{array}{l}\text { Fazekas white } \\
\text { matter }\end{array}$ & $\begin{array}{l}\text { Scheltens peri- } \\
\text { ventricular }\end{array}$ & $\begin{array}{l}\text { Scheltens white } \\
\text { matter }\end{array}$ & $\begin{array}{l}\text { Scheltens basal } \\
\text { ganglia }\end{array}$ \\
\hline mRS 0-1 & $1.46(\mathbf{0 . 0 0 3})$ & $4.43(\mathbf{0 . 0 3 3})$ & $0.63(\mathbf{0 . 0 0 1})$ & $0.83(0.061)$ & 1.01 (0.001) & $2.88(0.091)$ & $0.54(0.493)$ \\
\hline mRS 2-6 & 1.97 & 6.03 & 0.98 & 0.99 & 1.64 & 3.70 & 0.69 \\
\hline Survival & $1.68(<\mathbf{0 . 0 0 1})$ & $5.05(\mathbf{0 . 0 0 1})$ & $0.77(<\mathbf{0 . 0 0 1})$ & $0.90(\mathbf{0 . 0 2 3})$ & $1.26(<\mathbf{0 . 0 0 1})$ & 3.19 (0.008) & $0.60(0.36)$ \\
\hline Death & 2.40 & 7.62 & 1.29 & 1.11 & 2.25 & 4.56 & 0.81 \\
\hline $\begin{array}{l}\text { Recanalization } \\
\text { TIMI } 2+3\end{array}$ & $1.77(0.095)$ & 5.11 (0.011) & $0.85(0.109)$ & $0.92(0.168)$ & 1.37 (0.034) & $3.20(\mathbf{0 . 0 2})$ & $0.55(0.094)$ \\
\hline $\begin{array}{l}\text { Recanalization } \\
\text { TIMI } 0+1\end{array}$ & 2.05 & 6.99 & 1.01 & 1.04 & 1.78 & 4.31 & 0.90 \\
\hline sICH & $1.92(0.839)$ & $5.08(0.738)$ & $0.92(0.906)$ & $1.00(0.793)$ & $1.50(0.961)$ & $3.00(0.632)$ & $0.58(0.899)$ \\
\hline No sICH & 1.84 & 5.64 & 0.89 & 0.95 & 1.48 & 3.51 & 0.64 \\
\hline aICH & $1.90(0.67)$ & $5.79(0.767)$ & $0.94(0.524)$ & $0.96(0.94)$ & $1.47(0.959)$ & $3.39(0.777)$ & $0.93(0.081)$ \\
\hline No aICH & 1.82 & 5.56 & 0.87 & 0.95 & 1.48 & 3.53 & 0.55 \\
\hline
\end{tabular}

Bold values are statistically significant at $p<0.05$

subtraction angiography and recanalization TIMI 2 or 3 were identified as independent predictors for favorable clinical outcome (mRS 0-1) (Table 3). When Fazekas, Scheltens, Fazekas PV, Fazekas WM, Scheltens PV, Scheltens WM, and Scheltens BG scores were added separately into the model, Fazekas PV, Scheltens PV, and Fazekas turned out as independent predictors and replaced the factor age. Younger age, low NIHSS on admission, and good collaterals on arteriography independently predicted survival. In addition, Fazekas PV or Scheltens PV independently predicted survival.

\section{Predictors of recanalization}

Younger age and shorter time to treatment were independent predictors of recanalization after IAT (Table 3). When white matter lesion scores were added into the multivariate model, the Scheltens score turned out to be weakly predictive in exchange for the factor age.

\section{Predictors of hemorrhage}

Atrial fibrillation was identified as independent predictors for aICH, but not white matter lesion severity. A trend was seen for Fazekas $(p=0.265)$ and Scheltens BG $(p=0.152)$ scores. Asymptomatic hemorrhages were associated with the Scheltens BG $(p=0.036)$ score in univariate analysis, but showed only a trend in multivariate analysis $(p=0.138)$. Potentially due to the small sample size no predictors could be identified for SICH.

All these results were consistent with only minor changes in $p$ values when the 9 patients treated with combined IVT and IAT were excluded from the model or when only patients with MRI on the admission day were included in a separate analysis (data not shown).
Table 3 Multivariate logistic regression analysis: independent predictors for outcome, survival, and recanalization

\begin{tabular}{|c|c|c|c|}
\hline & $p$ & OR & $95 \% \mathrm{CI}$ \\
\hline \multicolumn{4}{|c|}{ Dependent variable outcome (mRS $0-1$ vs. $2-6$ ) } \\
\hline NIHSS & $<0.0001$ & 0.878 & $0.832-0.926$ \\
\hline Collaterals & 0.017 & 2.845 & $1.210-6.689$ \\
\hline Time to treatment & 0.039 & 0.996 & $0.992-1.000$ \\
\hline Diabetes mellitus & 0.026 & 0.274 & $0.088-0.856$ \\
\hline Recanalization & 0.021 & 2.542 & $1.148-5.629$ \\
\hline Fazekas PV & 0.007 & 0.569 & $0.379-0.856$ \\
\hline Fazekas & 0.021 & & \\
\hline Scheltens & 0.062 & & \\
\hline Scheltens PV & 0.011 & & \\
\hline
\end{tabular}

Dependent variable survival

\begin{tabular}{lccl} 
NIHSS & $<0.0001$ & 0.886 & $0.849-0.925$ \\
Collaterals & $<0.0001$ & 5.370 & $2.597-11.105$ \\
Age & 0.004 & 0.947 & $0.913-0.983$ \\
Fazekas PV & 0.018 & 0.550 & $0.335-0.904$ \\
\hline Fazekas & 0.107 & & \\
Scheltens & 0.067 & & \\
Scheltens PV & 0.014 & &
\end{tabular}

Dependent variable recanalization

$\begin{array}{llll}\text { Time to treatment } & 0.037 & 0.997 & 0.995-1.000\end{array}$

$\begin{array}{llll}\text { Scheltens } & 0.011 & 0.944 & 0.902-0.987\end{array}$

Dependent variable aICH

$\begin{array}{llll}\text { Atrial fibrillation } & 0.005 & 2.236 & 1.273-3.928\end{array}$

\section{Discussion}

The main finding of our study is that less severe periventricular white matter lesions in patients treated with IAT for 
acute stroke independently predicted favorable clinical outcome and survival. In addition, better recanalization occurred when there were less severe white matter lesions of the whole brain. The presence of white matter lesions in the basal ganglia region was associated with asymptomatic intracerebral bleedings in univariate but not in multivariate analysis.

White matter lesions have been identified as a risk factor for future strokes $[1,3,4]$ and as a predictor of unfavorable outcome in the spontaneous course of stroke [5, 19-23]. To date, the role of white matter lesions in patients treated with IVT for acute stroke is not clear. Using CT for assessment of white matter lesions, Ariës and co-workers [24] identified them as an independent predictor of poor functional outcome $(p=0.01$, OR $2.39,95 \%$ CI 1.21-4.72) in patients treated with IVT, while other authors did not find any association $[6,25]$. The poor sensitivity of CT to detect white matter lesions in comparison to MRI might account for these conflicting results in IVT studies.

Our study assessed white matter lesions with MRI and addressed their influence on outcome and survival after acute stroke treated with IAT. It shows that the severity of pre-existing white matter lesions is an important predictor for both functional outcome and survival. Furthermore, their regional distribution within the brain may be critical for outcome as well. Both the Fazekas and the Scheltens scores revealed white matter lesions in the periventricular region to be most predictive for clinical outcome and survival.

In addition, white matter lesions turned out to predict recanalization independently, though with a smaller odds ratio than for clinical outcome and survival. This interesting observation might be explained by the coexistence of small and large vessel disease. Patients with small vessel disease are more likely to also suffer from large vessel disease [26, 27].

In the multivariate analysis, white matter lesions compete with the factor age in predicting outcome and recanalization. This phenomenon has already been previously observed [7, 23]. Because age and hypertension are the most important predictors of white matter lesions, this finding is not unexpected. Potentially the severity of white matter lesions is a more precise predictor of outcome and survival than age, but this question has to be corroborated by further studies including more patients.

While one study with a mixed IVT and IAT [7] and two studies with an IVT population [6, 8] found white matter lesions to be independently predictive for sICH after IVT, one IVT study did not [24]. In our IAT series, there was no independent association of white matter lesions and sICH. White matter lesions were associated with asymptomatic ICH only in univariate analysis, but not after multivariable analysis and there was no association with symptomatic bleedings. This might be explained by the low number of sICH in our series and the differing definitions of sICH that are used in the different studies. However, the current practical implication might be that white matter lesions may not be considered as a contraindication to IAT. When symptomatic intracranial bleeding after IAT occurs, it is most likely not caused by white matter lesions.

Our study has several limitations. It is a single center non-randomized study with a potential selection bias. Another potential limitation is the visual scoring of white matter lesions, which is less accurate than volumetry [28]. On the other hand, volumetry of white matter lesions showed a nonlinear relationship between the Fazekas and Scheltens scores indicating that visual scoring is a valid method as well.

\section{Summary}

White matter lesions are a radiological sign easily visible on the widely available conventional MRI sequences. Our results show that white matter lesions in patients treated with IAT are independently predictive of outcome, mortality, and recanalization. Their severity is inversely correlated with favorable outcome, survival, and good recanalization. The severity of white matter lesions is also correlated with age and might be even a better prognostic indicator than age. Symptomatic intracranial bleeding after IAT are not associated with white matter lesions. Therefore, white matter lesions should not be considered as a contraindication against IAT.

Conflicts of interest No author has any conflict of interest.

\section{References}

1. Vermeer SE, Hollander M, van Dijk EJ, Hofman A, Koudstaal PJ, Breteler MM, Rotterdam Scan Study (2003) Silent brain infarcts and white matter lesions increase stroke risk in the general population: the Rotterdam Scan Study. Stroke 34:1126-1129

2. Vernooij MW, Ikram MA, Tanghe HL, Vincent AJ, Hofman A, Krestin GP, Niessen WJ, Breteler MM, van der Lugt A (2007) Incidental findings on brain MRI in the general population. N Engl J Med 357:1821-1828

3. Wen W, Sachdev PS (2004) Extent and distribution of white matter hyperintensities in stroke patients: the sydney stroke study. Stroke 35:2813-2819

4. Bokura H, Kobayashi S, Yamaguchi S, Iijima K, Nagai A, Toyoda G, Oguro H, Takahashi K (2006) Silent brain infarction and subcortical white matter lesions increase the risk of stroke and mortality: a prospective cohort study. J Stroke Cerebrovasc Dis 15:57-63

5. Fu JH, Lu CZ, Hong Z, Dong Q, Luo Y, Wong KS (2005) Extent of white matter lesions is related to acute subcortical infarcts and predicts further stroke risk in patients with first ever ischaemic stroke. J Neurol Neurosurg Psychiatr 76:793-796 
6. Palumbo V, Boulanger JM, Hill MD, Inzitari D, Buchan AM, CASES Investigators (2007) Leukoaraiosis and intracerebral hemorrhage after thrombolysis in acute stroke. Neurology 68:1020-1024

7. Neumann-Haefelin T, Hoelig S, Berkefeld J, Fiehler J, Gass A, Humpich M, Kastrup A, Kucinski T, Lecei O, Liebeskind DS, Rother J, Rosso C, Samson Y, Saver JL, Yan B, MR Stroke Group (2006) Leukoaraiosis is a risk factor for symptomatic intracerebral hemorrhage after thrombolysis for acute stroke. Stroke 37:2463-2466

8. Fiehler J, Siemonsen S, Thomalla G, Illies T, Kucinski T (2009) Combination of $\mathrm{T} 2 * \mathrm{~W}$ and FLAIR abnormalities for the prediction of parenchymal hematoma following thrombolytic therapy in 100 stroke patients. J Neuroimaging 19:311-316

9. Brott T, Adams HP Jr, Olinger CP, Marler JR, Barsan WG, Biller J, Spilker J, Holleran R, Eberle R, Hertzberg V et al (1989) Measurements of acute cerebral infarction: a clinical examination scale. Stroke 20:864-870

10. Arnold M, Schroth G, Nedeltchev K, Loher T, Remonda L, Stepper F, Sturzenegger M, Mattle HP (2002) Intra-arterial thrombolysis in 100 patients with acute stroke due to middle cerebral artery occlusion. Stroke 33:1828-1833

11. TIMI Study Group (1985) The Thrombolysis in Myocardial Infarction (TIMI) trial. Phase I findings. N Engl J Med 312:932_ 936

12. Kase CS, Furlan AJ, Wechsler LR, Higashida RT, Rowley HA, Hart RG, Molinari GF, Frederick LS, Roberts HC, Gebel JM, Sila CA, Schulz GA, Roberts RS, Gent M (2001) Cerebral hemorrhage after intra-arterial thrombolysis for ischemic stroke: the PROACT II trial. Neurology 57:1603-1610

13. Adams HP Jr, Bendixen BH, Kappelle LJ, Biller J, Love BB, Gordon DL, Marsh EE 3rd (1993) Classification of subtype of acute ischemic stroke. Definitions for use in a multicenter clinical trial. TOAST. Trial of Org 10,172 in acute stroke treatment. Stroke 24:35-41

14. European stroke initiative executive committee, EUSI writing committee, Olsen TS, Langhorne P, Diener HC, Hennerici M, Ferro J, Sivenius J, Wahlgren NG, Bath P (2003) European stroke initiative recommendations for stroke management-update 2003. Cerebrovasc Dis 16:311-337

15. European stroke organisation (ESO) executive committee, ESO writing committee (2008) Guidelines for management of ischaemic stroke and transient ischaemic attack 2008. Cerebrovasc Dis 25:457-507

16. van Swieten JC, Koudstaal PJ, Visser MC, Schouten HJ, van Gijn J (1988) Interobserver agreement for the assessment of handicap in stroke patients. Stroke. 19:604-607

17. Schmidt R, Fazekas F, Kleinert G, Offenbacher H, Gindl K, Payer F, Freidl W, Niederkorn K, Lechner H (1992) Magnetic resonance imaging signal hyperintensities in the deep and subcortical white matter. A comparative study between stroke patients and normal volunteers. Arch Neurol 49:825-827

18. Scheltens P, Barkhof F, Leys D, Pruvo JP, Nauta JJ, Vermersch P, Steinling M, Valk J (1993) A semiquantitative rating scale for the assessment of signal hyperintensities on magnetic resonance imaging. J Neurol Sci 114:7-12

19. Podgorska A, Hier DB, Pytlewski A, Czlonkowska A (2002) Leukoaraiosis and stroke outcome. J Stroke Cerebrovasc Dis 11:336-340

20. Liou LM, Chen CF, Guo YC, Cheng HL, Lee HL, Hsu JS, Lin RT, Lin HF (2010) Cerebral white matter hyperintensities predict functional stroke outcome. Cerebrovasc Dis 29:22-27

21. Koton S, Schwammenthal Y, Merzeliak O, Philips T, Tsabari R, Orion D, Dichtiar R, Tanne D (2009) Cerebral leukoaraiosis in patients with stroke or TIA: clinical correlates and 1 year outcome. Eur J Neurol 16:218-225

22. Kissela B, Lindsell CJ, Kleindorfer D, Alwell K, Moomaw CJ, Woo D, Flaherty ML, Air E, Broderick J, Tsevat J (2009) Clinical prediction of functional outcome after ischemic stroke: the surprising importance of periventricular white matter disease and race. Stroke 40:530-536

23. Arsava EM, Rahman R, Rosand J, Lu J, Smith EE, Rost NS, Singhal AB, Lev MH, Furie KL, Koroshetz WJ, Sorensen AG, Ay H (2009) Severity of leukoaraiosis correlates with clinical outcome after ischemic stroke. Neurology 72:1403-1410

24. Ariës MJ, Uyttenboogaart M, Vroomen PC, De Keyser J, Luijckx GJ (2010) tPA treatment for acute ischaemic stroke in patients with leukoaraiosis. Eur J Neurol 17:866-870

25. Demchuk AM, Khan F, Hill MD, Barber PA, Silver B, Patel S, Levine SR, NINDS rt-PA Stroke Study Group (2008) Importance of leukoaraiosis on $\mathrm{CT}$ for tissue plasminogen activator decision making: evaluation of the NINDS rt-PA Stroke Study. Cerebrovasc Dis $26: 120-125$

26. Hoshino A, Nakamura T, Enomoto S, Kawahito H, Kurata H, Nakahara Y, Ijichi T (2008) Clinical utility of evaluating intracranial artery stenosis and silent brain infarction to predict the presence of subclinical coronary artery disease in ischemic stroke patients. Intern Med 47:1775-1781

27. Ikram MA, Vernooij MW, Vrooman HA, Hofman A, Breteler MM (2009) Brain tissue volumes and small vessel disease in relation to the risk of mortality. Neurobiol Aging 30:450-456

28. van Straaten EC, Fazekas F, Rostrup E, Scheltens P, Schmidt R, Pantoni L, Inzitari D, Waldemar G, Erkinjuntti T, Mäntylä R, Wahlund LO, Barkhof F, LADIS group (2006) Impact of white matter hyperintensities scoring method on correlations with clinical data: the LADIS study. Stroke 37:836-840 